\title{
Phase diagram of selectively cross-linked block copolymers shows chemically microstructured gel
}

\author{
Alice von der Heydt ${ }^{1}$, a) and Annette Zippelius ${ }^{1,2}$ \\ 1) Institut für Theoretische Physik, Georg-August-Universität Göttingen, Friedrich-Hund-Platz 1, 37077 Göttingen, \\ Germany \\ ${ }^{2)}$ Max-Planck-Institut für Dynamik und Selbstorganisation, Am Faßberg 17, 3707r Göttingen, \\ Germany
}

(Dated: 17 August 2021)

We study analytically the intricate phase behavior of cross-linked $A B$ diblock copolymer melts, which can undergo two main phase transitions due to quenched random constraints: Gelation, i.e., spatially random localization of polymers forming a system-spanning cluster, is driven by increasing the number parameter $\mu$ of irreversible, type-selective cross-links between random pairs of $A$ blocks. Self-assembly into a periodic pattern of $A / B$-rich microdomains (microphase separation) is controlled by the $A B$ incompatibility $\chi$ inversely proportional to temperature. Our model aims to capture the system's essential microscopic features, including an ensemble of random networks that reflects spatial correlations at the instant of cross-linking. We identify suitable order parameters and derive a free-energy functional in the spirit of Landau theory that allows us to trace a phase diagram in the plane of $\mu$ and $\chi$. Selective cross-links promote microphase separation at higher critical temperatures than in uncross-linked diblock copolymer melts. Microphase separation in the liquid state facilitates gelation, giving rise to a novel gel state whose chemical composition density mirrors the periodic $A B$ pattern.

\section{INTRODUCTION}

Block copolymer melts are known to self-assemble into a variety of complex ordered microstructures ${ }^{1+3}$. Combining block copolymers and quenched disorder is considered promising, e.g., for the design of biomimetic materials with tunable micropatterns ${ }^{4}$. Irreversible, random cross-linking provides one method to introduce quenched disorder. On the one hand, sufficient cross-linking can stabilise the so-called microphases over a wide range of temperatures. On the other hand, cross-links in a chemically homogeneous gel can prevent ordered microphase separation, similarly as cross-links hinder macroscopic phase separation in a blend of homopolymers 5 .7. In general, there is a competition between ordering and random topological constraints due to cross-linking. Selective cross-links in biological heteropolymers provide cells with intriguing mechanisms of adaption: One example is peptidoglycan, also called murein, which is composed of sugars and amino-acids and forms a mesh-like wall around many bacteria's plasma membrane ${ }^{899}$.

For randomly and irreversibly cross-linked block copolymer melts, two phase-ordering mechanisms can be controlled independently: The incompatibility between the two chemical components of the block copolymer, usually quantified by the Flory-Huggins parameter $\chi$, controls the ordering transition in local chemical composition from a disordered (mixed) state to a periodic microstructure. The number, or the chemical potential, $\mu$, of random, irreversible, and here component-selective, cross-links controls gelation, a transition from a fluid (sol)

\footnotetext{
a) Electronic mail: heydt@theorie.physik.uni-goettingen.de
}

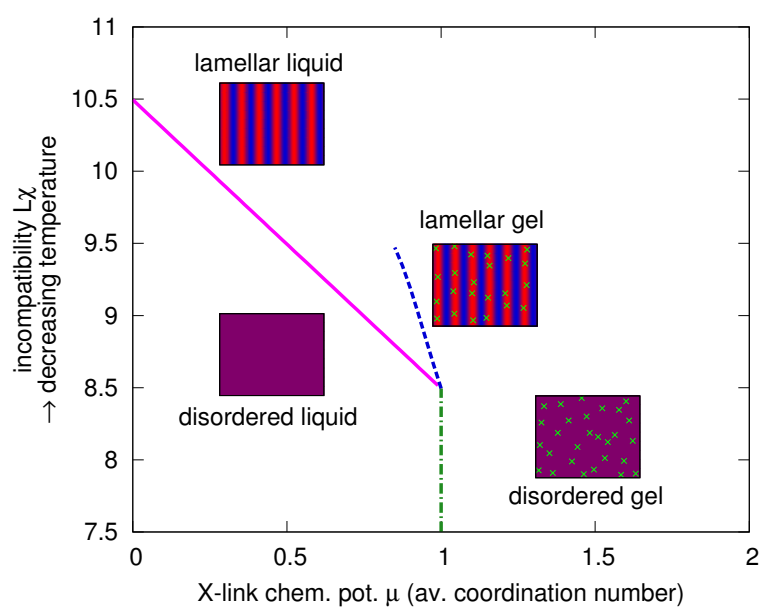

FIG. 1. Phase diagram in the plane of incompatibility, $\chi$, and chemical potential for the cross-links, $\mu$; four different phases are predicted: isotropic fluid, lamellar fluid, isotropic gel and lamellar gel.

to an amorphous solid (gel) 10 . This equilibrium state without long-range order, in which a fraction of polymers is localised at random positions, has been the subject of continued interest 11 .

Once the disordered sol state has undergone gelation, random localisation and irreversibility of the cross-links rule out the formation of an ordered microstructure. However, increasing the number of component-selective cross-links in the chemically ordered sol allows for a novel phase state - a microstructured gel. This microstructured gel is anisotropic and heterogeneous in chemical composition: In the simplest case of lamellae, segments of chemical component $A$ are localised randomly within 
each planar $A$-rich domain, but their concentration follows the periodic modulation of the lamellae in the third space dimension. The ordering is reminiscent of that in a smectic liquid crystal, except for the fact that within the planes, the particles in the gel cluster are not mobile as in a fluid but randomly localised as in an amorphous solid. The complete phase diagram, as obtained by the approach presented in the following, is shown in Fig. 1 in the plane of incompatibility, $\chi$, and cross-link chemical potential, $\mu$. Four different phases are present: an isotropic fluid, a lamellar (in general, microstructured) fluid, an isotropic gel and a microstructured gel. As alluded to above, the irreversible cross-linking considered here impedes transitions between the two gel phases. Which of the gel phases is actually observed, depends on the state of the melt at the instant of cross-linking.

In this paper, we discuss a mean-field theory in terms of two order-parameter fields: one for phase separation and one for random localisation. In this theory, the free energy takes the form of a Landau-Wilson expansion, which can be derived from a microscopic model. Alternatively, the Landau-Wilson free energy can be derived phenomenologically from symmetry arguments. We discuss both approaches, deferring most of the technical calculations to appendices. First, in Sec. III, we set up a microscopic model for randomly cross-linked block copolymers. Then, in Sec. III, we go on to discuss the collective fields or order parameters which follow naturally from the microscopic model and have an intuitive interpretation in terms of microphase ordering and gelation. In Sec. IV] we present the Landau-Wilson free energy used in the subsequent sections to obtain the phase diagram. In Sec. V] we discuss the lamellar fluid, and in Sec. VI. the instability to the lamellar gel. Conclusions and an outlook are presented in Sec. VII

\section{MODEL}

\section{A. Diblock copolymer melt}

The melt comprises $N$ monodisperse diblock copolymers with degree of polymerization $L$ confined to a volume $\tilde{V}$. The global segment density is $\varrho_{0}=N L / \tilde{V}$. We approximate the polymer contours $\boldsymbol{R}_{j}(s), j=1, \ldots, N$, $s \in[0,1]$, as Gaussian chains with radius of gyration $R_{G}=\sqrt{L b^{2} / 6}$ in three dimensions ( $b$ is the size of one statistical segment). For convenience, we choose units of energy such that $k_{B} T=1$ and measure all lengths in units of $R_{G}$, so that dimensionless Cartesian coordinates $r_{j, \nu}$ are defined according to $r_{j, \nu}=R_{j, \nu} / R_{G}$. The dimensionless system volume is $V=\tilde{V} / R_{G}^{3}$, and the global (dimensionless) segment density is denoted by $\varrho_{0}$. Accordingly, polymer conformations are governed by a quadratic effective potential

$$
\mathcal{H}_{N \mathrm{G}}=\frac{1}{4} \sum_{j=1}^{N} \int_{0}^{1} \mathrm{~d} s\left|\partial_{s} \boldsymbol{r}_{j}(s)\right|^{2},
$$

and in the following, $\mathcal{H}_{\mathrm{G}}$ is used to denote the singlechain potential.

For the inter-polymer interactions whose definitions follow, we adopt a semi-microscopic view, in which interaction shapes can be approximated by delta functions.

All polymer segments irrespective of their type interact via the excluded volume repulsion, which is of chiefly entropic origin and thus can be written as

$$
\mathcal{H}_{\mathrm{ev}}=\frac{\kappa N L}{4 \varrho_{0}^{2}} \int \mathrm{d}^{3} r \varrho(\boldsymbol{r}) \varrho(\boldsymbol{r})
$$

where $\kappa>0$ is the (dimensionless) compression modulus, and $\varrho(\boldsymbol{r}):=\frac{L}{V} \sum_{j=1}^{N} \int_{0}^{1} \mathrm{~d} s \delta\left(\boldsymbol{r}-\boldsymbol{r}_{j}(s)\right)$ is the total density.

Since the van-der-Waals attraction between chemically different polymer segments is weaker than between equal ones, there is a net repulsion or incompatibility between type $A$ and $B$. In each diblock copolymer, the block of type $A$ comprises a fraction $f \in[0,1]$ of the chain length, and the block of type $B$ a fraction $1-f$. A binary variable $q(s)$ records the type at contour position $s$ via

$$
q(s):= \begin{cases}+1, & s \text { is of type } A, \\ -1, & s \text { is of type } B\end{cases}
$$

(due to monodispersity, $q(s)$ does not depend on the polymer index $j$ ). The average value $q=2 f-1$, related to the $A$ fraction $f$, quantifies the global excess of $A$ segments. Expressed by the local imbalance or $A$ excess density

$$
\sigma(\boldsymbol{r}):=\frac{L}{V} \sum_{j=1}^{N} \int_{0}^{1} \mathrm{~d} s[q(s)-q] \delta\left(\boldsymbol{r}-\boldsymbol{r}_{j}(s)\right),
$$

the incompatibility interaction between $A$ and $B$ segments takes the form

$$
\mathcal{H}_{\chi}=-\frac{\chi N L}{4 \varrho_{0}^{2}} \int \mathrm{d}^{3} r \sigma(\boldsymbol{r}) \sigma(\boldsymbol{r})
$$

where $\chi$ denotes the incompatibility (Flory) parameter, which is assumed to be positive.

\section{B. Random, irreversible, type-selective cross-links}

Motivated by experiment 12 , we consider a synthesis scheme, which incorporates cross-linkers selectively, here only into the $A$ blocks of the copolymers, cf. Fig. 22 Randomly chosen pairs of $A$ blocks, $j_{m}, j_{m}^{\prime} \in$ $\{1,2, \ldots, N\}$, are permanently cross-linked at random arc-lengths, $s_{m}, s_{m}^{\prime} \in[0, f]$, so that one configuration (instance) of $M$ cross-links is specified by the set of pairs 

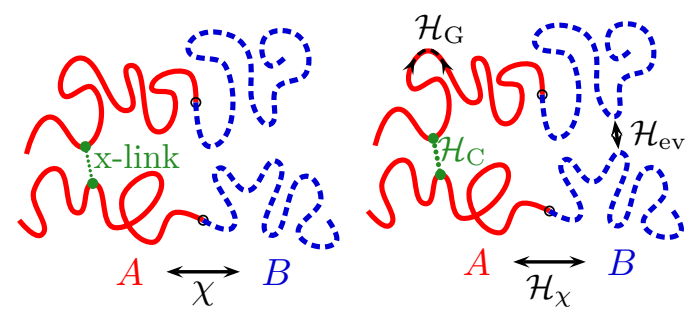

FIG. 2. Selective cross-link between the $A$ blocks of two diblock copolymers and interactions considered in the model.

$\mathcal{C}:=\left\{\left(\left(j_{m}, s_{m}\right),\left(j_{m}^{\prime}, s_{m}^{\prime}\right)\right)\right\}_{m=1}^{M}$. The cross-links are modeled as entropic, harmonic springs of (tunable) constant $1 /\left(2 a_{c}^{2}\right)$,

$$
\mathcal{H}_{c}=\frac{1}{4 a_{c}^{2}} \sum_{m=1}^{M}\left|\boldsymbol{r}_{j_{m}}\left(s_{m}\right)-\boldsymbol{r}_{j_{m}^{\prime}}\left(s_{m}^{\prime}\right)\right|^{2} .
$$

Pursuing a statistical mechanical approach, we aim at computing the free energy of the model specified by the total Hamiltonian

$$
\mathcal{H}\left\{q(s), \boldsymbol{r}_{j}(s)\right\}=\mathcal{H}_{N G}+\mathcal{H}_{\mathrm{ev}}+\mathcal{H}_{\chi}+\mathcal{H}_{c}
$$

the parts of which are sketched in the right panel of Fig. 2. Rather than in the absolute free energy, we are interested in the free-energy difference between the cross-linked diblock copolymer melt with $A B$ incompatibility and a disordered, single-type (homo)polymer melt with uniform densities and with no interactions but intra-chain connectivity. Therefore, we normalise the canonical partition functions with the partition function of a homogeneous melt of $N$ non-interacting Gaussian chains,

$$
\mathcal{Z}(\mathcal{C})=\frac{\int \mathcal{D}\left[\boldsymbol{r}_{j}(s)\right] \mathrm{e}^{-\mathcal{H}\left\{q(s), \boldsymbol{r}_{j}(s)\right\}}}{\int \mathcal{D}\left[\boldsymbol{r}_{j}(s)\right] \mathrm{e}^{-\mathcal{H}_{N G}\left\{\boldsymbol{r}_{j}(s)\right\}}} .
$$

Herein, $\mathcal{D}\left[\boldsymbol{r}_{j}(s)\right]$ denotes the measure of functional integrations over the polymer configurations $\boldsymbol{r}_{j}(s), j=$ $1, \ldots, N, s \in[0,1]$. Since we consider a random ensemble of irreversible cross-links, the disorder due to crosslinks is quenched, hence the quantity to be reasonably disorder-averaged is the free energy, i.e., $-\ln \mathcal{Z}(\mathcal{C})$, where $\mathcal{Z}(\mathcal{C})$ is the canonical partition function of cross-link configuration $\mathcal{C}$.

We choose a grand-canonical cross-link ensemble, in which instead of the number of cross-links, $M$, their chemical potential $\mu>0$ is fixed. All cross-links are assumed to be formed simultaneously and instantaneously in the liquid phase - a process which is well modeled by the Deam-Edwards distribution 13

$$
\mathcal{P}_{\mathrm{DE}}(\mathcal{C} ; M) \propto \mathcal{Z}(\mathcal{C}) \frac{\mu^{M}}{M !} .
$$

From the cross-link chemical potential $\mu$, the average polymer coordination number in the network follows as
$\mu=2[M]_{\mathcal{P}_{\mathrm{DE}}} / N$. Via including the partition function at preparation, the Deam-Edwards distribution 'measures' the loss in configurational entropy due to the cross-link constraints and thereby accounts for spatial correlations in the uncross-linked melt. Also, the distribution allows to choose the preparation state, in which cross-linking is performed, to be different from the measurement state, i.e., the state in which the network is probed in experiment. Here, for a first overview, we assume the preparation and the measurement state to be the same.

With the cross-link distribution of Eq. (9), the specification of the model is complete, and we can proceed to derive the disorder-averaged free energy from first principles. The resulting saddle-point equations for the order parameters for phase separation (ordering), gelation, and possibly mixed transitions, then have to be solved self-consistently. The basic steps of this procedure, eventually yielding a free energy of Ginzburg-Landau-Wilson form, are sketched in the appendix. In the main text, we will consider this Ginzburg-Landau-Wilson free energy only, since its essential features can be derived from general symmetry principles, without the use of replicas. This requires a careful discussion of the order parameters, which is the focus of the next section.

\section{ORDER PARAMETERS}

Phase separation into $A / B$-rich domains is signaled by spatial modulations of the imbalance density

$$
\sigma(\boldsymbol{k})=\frac{L}{V} \sum_{j=1}^{N} \int_{0}^{1} \mathrm{~d} s(q(s)-q)\left\langle\mathrm{e}^{i \boldsymbol{k} \cdot \boldsymbol{r}_{j}(s)}\right\rangle
$$

cf. Eq. (3) for the type variable $q(s)$, where $\langle\cdot\rangle$ denotes the thermal average with the total Hamiltonian from Eq. (7) for a fixed cross-link configuration. Here, we will mainly address symmetric $A B$ diblock copolymers, i.e., $A$-fraction $f=1 / 2$ or $q=0$, in which a lamellar state arises, the prototype of an ordered microstructure. The imbalance density in this state can be parametrized by a one-dimensional, single-mode distribution in space,

$$
\sigma(\boldsymbol{x})=\sigma_{0} \cos \left(\boldsymbol{q}_{0} \cdot \boldsymbol{x}+\phi\right)
$$

characterized by the amplitude $\sigma_{0}$, the wavevector $\boldsymbol{q}_{0}$ and a phase $\phi$. In fact, there is a manifold of symmetryrelated states defined by $\phi$ and the direction of $\boldsymbol{q}_{0}$, and we are free to choose one of them, viz., $\phi=0$ and $\boldsymbol{q}_{0}=$ $q_{0} \boldsymbol{e}_{1}$. The corresponding probability density distribution $w_{(j, s) \text { fluid }}(\boldsymbol{x})=\left\langle\delta\left(\boldsymbol{x}-\boldsymbol{r}_{j}(s)\right)\right\rangle$ of a single mobile (fluid) $A$ segment is sketched in the left panel of Fig. 3 .

An $A$ segment $(j, s)$ in the gel state is localised at a random position $\boldsymbol{a}_{j}(s)$. In a Gaussian localisation model, the probability distribution is sharply peaked around $\boldsymbol{a}_{j}(s)$

$$
w_{(j, s) \text { loc. }}(\boldsymbol{x})=\left\langle\delta\left(\boldsymbol{x}-\boldsymbol{r}_{j}(s)\right)\right\rangle \propto \mathrm{e}^{-\left(\boldsymbol{x}-\boldsymbol{a}_{j}(s)\right)^{2} /\left(2 \xi^{2}\right)},
$$



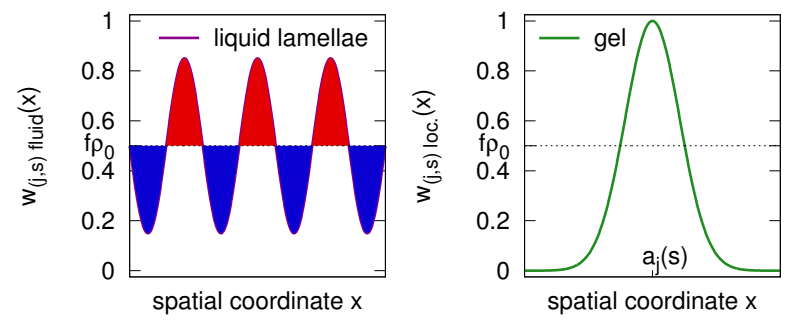

FIG. 3. One-dimensional, single- $A$ probability density distribution in the fluid lamellar (left) and in the gel (right) state.

as shown schematically in the right panel of Fig. 3 The extent of spatial localisation is measured by the localisation length $\xi$. Since the positions are random, the average density vanishes in the thermodynamic limit. The macroscopic observable able to detect random localisation with a minimal number of arguments is

$$
\Omega(\boldsymbol{k}, \boldsymbol{p})=\frac{1}{N f} \sum_{j=1}^{N} \int_{0}^{f} \mathrm{~d} s\left\langle\mathrm{e}^{i \boldsymbol{k} \cdot \boldsymbol{r}_{j}(s)}\right\rangle\left\langle\mathrm{e}^{i \boldsymbol{p} \cdot \boldsymbol{r}_{j}(s)}\right\rangle .
$$

Due to the $A$-selective cross-links or topological constraints, it suffices to sum over the $A$ segments.

Since phase separation and gelation are controlled independently, we expect to find four different phases:

- isotropic liquid • isotropic gel

- lamellar liquid • lamellar gel

In the isotropic fluid, both order parameters vanish. In the isotropic gel, the Gaussian mode ${ }^{10}$ predicts

$$
\begin{aligned}
\sigma(\boldsymbol{k}) & =0 \text { and } \\
\Omega_{\mathrm{iso}}(\boldsymbol{k}, \boldsymbol{p}) & =\underbrace{Q \delta_{\boldsymbol{k}+\boldsymbol{p}, 0} e^{-k^{2} \xi^{2} / 2}}_{\delta \Omega_{\mathrm{iso}}(\boldsymbol{k}, \boldsymbol{p})}+(1-Q) \delta_{\boldsymbol{k}, 0} \delta_{\boldsymbol{p}, 0 .}
\end{aligned}
$$

The finite fraction of localised $A$-blocks is denoted by $Q$, and macroscopic translational invariance of the amorphous gel requires $\boldsymbol{k}+\boldsymbol{p}=0$ for the gel part $\delta \Omega_{\text {iso }}$.

In the lamellar fluid, both order parameters are nonzero, even though the particles are not localised in the lamellar fluid, but exhibit periodic density modulations. Consistent with Eqs. (11) and (13), we can deduce the following minimal parametrizations of the order parameters in the lamellar fluid:

$$
\begin{aligned}
\sigma_{\operatorname{lam}}(\boldsymbol{k}) & =\sigma_{0}\left(\delta_{\boldsymbol{k}, \boldsymbol{q}_{0}}+\delta_{\boldsymbol{k},-\boldsymbol{q}_{0}}\right), \\
\Omega_{\mathrm{lam}}(\boldsymbol{k}, \boldsymbol{p}) & \propto \sigma_{0}^{2}\left(\delta_{\boldsymbol{k}, \boldsymbol{q}_{0}}+\delta_{\boldsymbol{k},-\boldsymbol{q}_{0}}\right)\left(\delta_{\boldsymbol{p}, \boldsymbol{q}_{0}}+\delta_{\boldsymbol{p},-\boldsymbol{q}_{0}}\right) .
\end{aligned}
$$

In the lamellar gel, we expect the order parameter Eq. 13 to reflect both ordering and localisation: There is the lamellar-fluid contribution, Eq. (17), and a true gel contribution $\delta \Omega(\boldsymbol{k}, \boldsymbol{p})$, which accounts for localisation of a fraction $Q$ of polymers. To reflect the lamellar order imprinted in the gel, $\delta \Omega$ must again split up and feature, in addition to the isotropic gel $\delta \Omega_{\text {iso }}$, Eq. (14), a novel part. Inserting the Gaussian localisation model, we find

$$
\delta \Omega(\boldsymbol{k}, \boldsymbol{p})=\frac{Q}{N f} \sum_{j=1}^{N} \int_{0}^{f} \mathrm{~d} s \mathrm{e}^{i(\boldsymbol{k}+\boldsymbol{p}) \cdot \boldsymbol{a}_{j}(s)} \mathrm{e}^{-\left(k^{2}+p^{2}\right) \xi^{2} / 2} .
$$

To perform the spatial average over (partially) random localisation centres $\left\{\boldsymbol{a}_{j}(s)\right\}$ of $A$, we need to know their probability distribution. In a mean-field picture, we can assume that all $\boldsymbol{a}_{j}(s)$ are independently, identically distributed. The single-centre probability distribution

$$
p(\boldsymbol{a})=\frac{1}{V}+\frac{\sigma_{0}}{\varrho_{0}} \cos \left(\boldsymbol{q}_{0} \cdot \boldsymbol{a}\right)
$$

displays a uniform part as in the isotropic gel state and a periodic modulation in the direction of $\boldsymbol{q}_{0}$, generated by the pattern of $A$-rich microdomains and proportional to $\sigma_{0}(c f .$, the single- $A$ density distribution in the left panel of Fig. 3. but recall that $p(\boldsymbol{a})$ is the mean-field distribution of a localisation position.) With this distribution, the lamellar gel part of the order parameter becomes

$$
\begin{aligned}
& \delta \Omega(\boldsymbol{k}, \boldsymbol{p}) \\
& =Q\left(\delta_{\boldsymbol{k}+\boldsymbol{p}, \mathbf{0}}+\frac{\sigma_{0}}{\varrho_{0}}\left(\delta_{\boldsymbol{k}+\boldsymbol{p}, \boldsymbol{q}_{0}}+\delta_{\boldsymbol{k}+\boldsymbol{p},-\boldsymbol{q}_{0}}\right)\right) \mathrm{e}^{-\left(k^{2}+p^{2}\right) \xi^{2} / 2} .
\end{aligned}
$$

A more elaborate theory should account for an anisotropic degree of localisation, i.e., allow for different localisation lengths within the plane of a lamellar domain and perpendicular to it. Here, to discuss the instabilities toward the different phases, there is no need to detail the form of localisation, so we leave this refined ansatz for future work. In the next section, we discuss the LandauWilson free energy in terms of the two order parameters introduced above, $\sigma(\boldsymbol{k})$ and $\Omega(\boldsymbol{k}, \boldsymbol{p})$.

\section{LANDAU-WILSON FREE ENERGY}

As mentioned above, the Landau-Wilson free energy can be derived completely from the microscopic model, and we summarize this calculation in Appendix $\mathrm{A}$. In this part, we just quote the result which - apart from the precise form of the vertices - follows also from symmetry principles. As needed, we use symbolic notation for the vertices. Also for reasons of transparency, we restrict the consideration to the $A \leftrightharpoons B$ exchange-symmetric diblock melt, i.e., $A$-fraction $f=1 / 2$.

We decompose the total free energy into three parts:

$$
F[\sigma, \Omega]=F_{\sigma}[\sigma]+F_{\Omega}[\Omega]+F_{\sigma \Omega}[\sigma, \Omega] .
$$

The first one, $F_{\sigma}$, accounts for lamellar phase separation and displays the standard form for symmetric $A B$ 


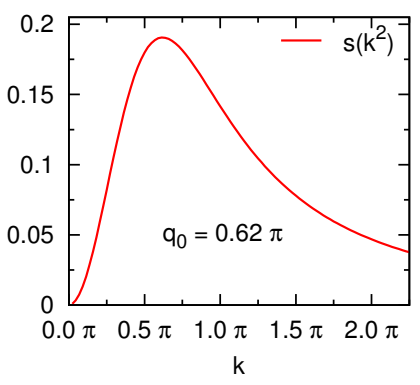

FIG. 4. Correlation $s\left(k^{2}\right)$ for symmetric diblocks.

block copolymers (here, we abbreviate $\sigma(\boldsymbol{k})$ by $\sigma_{\boldsymbol{k}}$ ):

$$
\begin{aligned}
F_{\sigma}[\sigma]= & \frac{L \chi_{\mu}}{4 \varrho_{0}^{2}} \sum_{\boldsymbol{k} \neq \mathbf{0}}\left(1-L \chi_{\mu} \frac{s\left(k^{2}\right)}{2}\right) \sigma_{\boldsymbol{k}} \sigma_{-\boldsymbol{k}} \\
& +\left[\frac{L \chi_{\mu}}{\varrho_{0}}\right]_{\boldsymbol{k}_{1}, \boldsymbol{k}_{2}, \boldsymbol{k}_{3}}^{4} s_{\sigma^{4}}\left(\left\{\boldsymbol{k}_{l}\right\},\left\{\sigma_{\boldsymbol{k}_{l}}, \sigma_{-\sum_{l=1}^{3} \boldsymbol{k}_{l}}\right\}\right)
\end{aligned}
$$

with the cross-link-rescaled incompatibility

$$
L \chi_{\mu}:=L \chi+2 \mu .
$$

The correlation function in the quadratic term is

$$
\begin{aligned}
s\left(k^{2}\right) & =\iint_{0}^{1} \mathrm{~d} s_{1} \mathrm{~d} s_{2} q\left(s_{1}\right) q\left(s_{2}\right)\left\langle\mathrm{e}^{-i \boldsymbol{k} \cdot\left(\boldsymbol{r}\left(s_{1}\right)-\boldsymbol{r}\left(s_{2}\right)\right)}\right\rangle_{\mathcal{H}_{\mathrm{G}}} \\
& =4 d_{1 / 2}\left(k^{2}\right)-d_{1}\left(k^{2}\right),
\end{aligned}
$$

with the Debye function $d_{a}\left(k^{2}\right):=2\left(\mathrm{e}^{-a k^{2}}-1+a k^{2}\right) / k^{4}$. It exhibits the known feature of a maximum at a finite wavenumber $q_{0}, c f .$, Fig. 4, corresponding to a lamellar wavelength of $3.2 R_{G}$ at the onset of microphase separation. Thereby, $q_{0}$ is the instability mode, since upon increasing $\chi$, the homogeneously mixed phase with $\sigma_{\boldsymbol{k}}=0$ becomes unstable when the first coefficient of the quadratic term (in the mixed state positive) vanishes: $2-L \chi_{\mu, c} s\left(q_{0}^{2}\right)=0$. At this point, cross-links influence the $A B$ order only via the rescaled incompatibility Eq. 23): For the unconstrained melt of diblocks, microphase separation occurs at $L \chi_{c}=2 / s\left(q_{0}^{2}\right)$, whereas selective cross-linking shifts this transition to lower $\chi$ (or higher temperatures):

$$
L \chi_{c}=\frac{2}{s\left(q_{0}^{2}\right)}-2 \mu
$$

This effect is known $14 \sqrt{16}$ and intuitively comprehensible: Selective cross-links give rise to chemical ordering via the formation of $A$-rich regions and thus favour $A B$ phase separation. Finally, the vertices of the fourth-order term, summarized by $s_{\sigma^{4}}$ in the constrained sum $\sum^{\prime}$, can be computed as correlation functions of the Gaussian theory explicitly defined in Appendix B.

The second term in Eq. 21), accounts for the gel transition in the absence of phase separation. We restrict ourselves here to the minimal complexity of the order parameter, $\Omega(\boldsymbol{k}, \boldsymbol{p})$, defined as the second moment of the local density in Eq. (13). A complete theory of gelation requires all moments $\frac{10}{10}$. However, we will see shortly that only the second moment couples to the order parameter for phase separation, so that we can simplify the free energy considerably:

$$
\begin{aligned}
& F_{\Omega}[\Omega]= \\
& \frac{\mu}{2} \sum_{\boldsymbol{k}, \boldsymbol{p} \neq 0}\left(1-4 \mu d_{1 / 2}\left(k^{2}+p^{2}\right)\right) \Omega(\boldsymbol{k}, \boldsymbol{p}) \Omega(-\boldsymbol{k},-\boldsymbol{p}) \\
& -\frac{\mu^{3}}{6} \sum_{\boldsymbol{k}_{1}, \boldsymbol{k}_{2} \neq 0} \sum_{\boldsymbol{p}_{1}, \boldsymbol{p}_{2} \neq 0} s_{\Omega^{3}}\left(\boldsymbol{k}_{1}, \boldsymbol{k}_{2} ; \boldsymbol{p}_{1}, \boldsymbol{p}_{2}\right) \\
& \quad \times \Omega\left(\boldsymbol{k}_{1}, \boldsymbol{k}_{2}\right) \Omega\left(\boldsymbol{p}_{1}, \boldsymbol{p}_{2}\right) \Omega\left(-\boldsymbol{k}_{1}-\boldsymbol{p}_{1},-\boldsymbol{k}_{2}-\boldsymbol{p}_{2}\right)
\end{aligned}
$$

Here, $s_{\Omega^{3}}$ is a vertex function (or constant, if evaluated at wave numbers zero), which can be computed from the microscopic model. The fluid phase (sol) with $\Omega=0$ becomes unstable to the formation of an isotropic gel when the concentration of cross-links is sufficiently high so that $1-\mu d_{1 / 2}\left(k^{2}+p^{2}\right)=0$. The maximum of the correlation function $d_{1 / 2}\left(k^{2}+p^{2}\right)$ occurs at $k^{2}+p^{2}=0$, so that without lamellar order, the gel transition occurs at $\mu_{c}=1$, independent of the incompatibility $\chi$. This transition is marked by the vertical line in Fig. 1 1 . The continuous growth of the gel fraction $Q$ as a function of $\mu-\mu_{c}$ is known already from the percolation description by Flory and Stockmayer ${ }^{17 / 18}$ and can actually be computed by inserting the isotropic-gel ansatz for $\Omega$ into Eq. (26); the interested reader may consult, e.g. $\frac{10|19| 20}{\text {. }}$

The third term in Eq. 21) reflects the coupling of the two order parameters, accounting for phase separation and gelation. The lowest order terms allowed by symmetry are explicitly given by

$$
\begin{aligned}
& F_{\sigma \Omega}[\sigma, \Omega]= \\
& =-\frac{\left(L \chi_{\mu}\right)^{2} \mu}{8 \varrho_{0}^{2}} \sum_{\boldsymbol{k}, \boldsymbol{p} \neq 0} \sigma_{\boldsymbol{k}} \sigma_{\boldsymbol{p}} \Omega(-\boldsymbol{k},-\boldsymbol{p}) s_{\sigma^{2} \Omega}\left(k^{2}, p^{2}\right) \\
& -\frac{L \chi_{\mu} \mu^{2}}{4 \varrho_{0}} \sum_{\boldsymbol{k}, \boldsymbol{p}, \boldsymbol{q} \neq 0} \sigma_{\boldsymbol{q}} \Omega(\boldsymbol{k}, \boldsymbol{p}) \Omega(-\boldsymbol{k}-\boldsymbol{q},-\boldsymbol{p}) s_{\sigma \Omega^{2}}(\boldsymbol{q} ;(\boldsymbol{k}, \boldsymbol{p})) .
\end{aligned}
$$

In a phenomenological approach, the correlations $s_{\sigma^{2} \Omega}$ and $s_{\sigma \Omega^{2}}$ are constants, whereas in the microscopic approach these correlations can be computed from first principles, as shown in the appendix. In the following sections, we are going to analyze the free energy and discuss the lamellar state as well as the instability towards a lamellar gel.

\section{LAMELLAR FLUID}

As discussed above, the chemically homogeneous melt becomes unstable to microphase separation at $2-$ $L \chi_{\mu_{c}} s\left(q_{0}^{2}\right)=0$. In the vicinity of the transition line, the 
amplitude of the composition modulation is expected to be small and hence appropriately described by a LandauWilson expansion of the free-energy functional. Assuming a lamellar state [Eq. [17]], the free energy becomes

$$
\begin{aligned}
& F_{\text {lam }}[\sigma, \Omega]= \\
& \frac{L \chi_{\mu}}{4 \varrho_{0}^{2}}\left(1-L \chi_{\mu} \frac{s\left(q_{0}^{2}\right)}{2}\right) \sigma_{0}^{2}+c_{1} \sigma_{0}^{4} \\
& +\frac{\mu}{2} \sum_{\boldsymbol{k}, \boldsymbol{p} \neq 0}\left(1-4 \mu d_{1 / 2}\left(k^{2}+p^{2}\right)\right) \Omega(\boldsymbol{k}, \boldsymbol{p}) \Omega(-\boldsymbol{k},-\boldsymbol{p}) \\
& -2 c_{3} \sigma_{0}^{2}\left(\Omega\left(\boldsymbol{q}_{0}, \boldsymbol{q}_{0}\right)+\Omega\left(\boldsymbol{q}_{0},-\boldsymbol{q}_{0}\right)\right)+\mathcal{O}\left(\Omega^{3}, \sigma \Omega^{2}\right)
\end{aligned}
$$

Here $c_{1}$ and $c_{3}$ abbreviate the vertex functions $s_{\sigma^{4}}$ and $s_{\sigma^{2} \Omega}$, evaluated at the lamellar wavevectors $\pm \boldsymbol{q}_{0}$.

Variation of $F_{\text {lam }}$ with respect to the order-parameter fields yields saddle-point equations, which inter alia show that the gel order parameter $\Omega$ assumes a nontrivial value as a function of the amplitude $\sigma_{0}$ even in the fluid state. The complete theory, including all moments of $\Omega$, cf. Appendix A, shows that to lowest order in the amplitude $\sigma_{0}$, indeed only the second moment is affected, as announced before Eq. (26). For a lamellar microphase, the saddle-point equation, see also Eq. (B3), reads

$$
\Omega_{\mathrm{lam}}(\boldsymbol{k}, \boldsymbol{p})=\frac{c_{3} \sigma_{0}^{2}\left(\delta_{\boldsymbol{k}, \boldsymbol{q}_{0}}+\delta_{\boldsymbol{k},-\boldsymbol{q}_{0}}\right)\left(\delta_{\boldsymbol{p}, \boldsymbol{q}_{0}}+\delta_{\boldsymbol{p},-\boldsymbol{q}_{0}}\right)}{\mu\left(1-4 \mu d_{1 / 2}\left(k^{2}+p^{2}\right)\right)},
$$

valid for $\mu<1$. This implies a periodic modulation of the second moment of the local density of $A$ segments, as anticipated in Eq. (17). Not written out explicitly here, generalized compression modes $\kappa \varrho_{\boldsymbol{k}}$ of the simple density are on the saddle-point level also quadratic in $\sigma_{\boldsymbol{k}}$ in the symmetric case. However, the dominant wave number of this modulation is $2 q_{0}$, which can be explained with the system's tendency to compress both $A$ - and $B$-rich regions.

Substituting these results back into the equation for the lamellar amplitude $\sigma_{0}$ and solving for $\sigma_{0} \neq 0$, we find

$$
\sigma_{0}^{2} \propto 1-L \chi_{\mu} \frac{s\left(q_{0}^{2}\right)}{2}
$$

close to the transition. As expected, the lamellar amplitude grows continuously like a square root as a function of the distance to the critical point - characteristic of a mean-field theory. For microphase separation without coupling to another transition, this result was first derived by Leibler ${ }^{1}$. Later, in Ref. ${ }^{21}$, it was shown that fluctuations induce a first-order transition in the same universality class as the Brazovskiǔ mode ${ }^{22 \mid 23}$.

Interestingly, the contribution of the second moment of $\Omega$ causes an increase in the amplitude compared to a diblock melt without cross-links. Due to the wave-number dependence of the denominator of the expression for $\sigma_{0}$ (omitted in Eq. (30)), also the lamellar wave number generally changes continuously from $q_{0}$ when the amplitude increases.

We summarize the main results obtained so far: In the fluid state, a phase transition to a lamellar state occurs at a critical value of the incompatibility. Cross-linking causes a pre-ordering which facilitates phase separation, so that the critical incompatibility depends on the degree of cross-linking. Microphase separation and crosslinking are coupled already in this state which has not yet undergone gelation. Furthermore, within the lamellar phase, the gel order parameter is nonzero, even though the monomers are not strictly localised, but follow a periodic probability density.

\section{LAMELLAR GEL}

What happens, if we increase the number of cross-links in the lamellar fluid? At which cross-link density does gelation set in, what is the structure of the resulting gel, and what remains of the microstructure? To answer these questions, we test the stability towards gelation by inserting small deviations $\delta \Omega$ of the gel order parameter from its value in the lamellar fluid, Eq. (29), viz.,

$$
\Omega\left(\boldsymbol{k}_{1}, \boldsymbol{k}_{2}\right)=\Omega_{\mathrm{lam}}\left(\boldsymbol{k}_{1}, \boldsymbol{k}_{2}\right)+\delta \Omega\left(\boldsymbol{k}_{1}, \boldsymbol{k}_{2}\right),
$$

into the free-energy functional and expanding the freeenergy difference

$$
\Delta F=F[\sigma, \Omega]-F_{\text {lam }}[\sigma, \Omega]
$$

up to quadratic order in $\delta \Omega$. In doing this, we insert the form of the novel component of the gel order parameter dictated by the discrete translation symmetry of the lamellar composition pattern, $c f .$, Eq. 20p.

As suggested by the form of the contributions to the gel order parameter discussed in Sec. III] we decompose the deviation (vector) $\boldsymbol{\delta} \Omega$ into orthogonal components that represent isotropic, homogeneous, respectively sinusoidal density of localised $A$ segments:

$$
\begin{aligned}
& \boldsymbol{\delta} \Omega\left(\boldsymbol{k}_{1}, \boldsymbol{k}_{2}\right)= \\
& \delta \Omega^{(\mathrm{i})} \delta_{\boldsymbol{k}_{1}+\boldsymbol{k}_{2}, \mathbf{0}}+\delta \Omega^{(+)} \delta_{\boldsymbol{k}_{1}+\boldsymbol{k}_{2},+\boldsymbol{q}_{0}}+\delta \Omega^{(-)} \delta_{\boldsymbol{k}_{1}+\boldsymbol{k}_{2},-\boldsymbol{q}_{0}} .
\end{aligned}
$$

Since the lamellar modulation affects one space dimension (defined by $\boldsymbol{q}_{0}$, labeled 1) only, we focus on the component $k:=k_{1,1}$ of the variable wavevector $\boldsymbol{k}_{1}$. Additionally, only one of the wave numbers $k_{1,1}$ and $p_{1,1}$ is a free parameter, called $k$, due to translational invariance of the conformational average, $c f$. , Eq. (27). The resulting quadratic form

$$
\Delta F=\sum_{m, n \in\{\mathrm{i},+,-\}} G^{(m, n)}(k) \delta \Omega^{(m)} \delta \Omega^{(n)},
$$

parametrized by $k$ can be further simplified by taking the limit $k \rightarrow 0$, anticipating the gel instability to set in with a small critical wave number (without lamellar order, the gel instability occurs at wave number $k_{\text {gel }}=0$ ). Coupling of these matrices for each $k$ and thus a more complicated diagonalisation would arise but for $k \geq 2 q_{0}$. Still, the description includes the lamellar wave number $q_{0}$, which 
can be reasonably assumed to be much larger than the gel instability wave number. In this limit, the $(+)$ and (-) wave contributions collapse to a single one, and the problem is reduced to computing the eigenvalues of the symmetric $2 \times 2$ instability matrix

$$
G=\left(\begin{array}{cc}
1-\mu & -\mu_{\sigma_{0}} s_{\sigma \Omega^{2}}\left(q_{0}^{2}\right) \\
-\mu_{\sigma_{0}} s_{\sigma \Omega^{2}}\left(q_{0}^{2}\right) & 2\left(1-4 \mu d_{1 / 2}\left(q_{0}^{2}\right)\right)
\end{array}\right)
$$

with the amplitude-dependent quantity

$$
\mu_{\sigma_{0}}:=\frac{\mu L \chi_{\mu} \sigma_{0}}{2 \varrho_{0}}
$$

The mixed vertex $s_{\sigma \Omega^{2}}$, Eq. (B7), evaluated at $k=0$ is

$$
\begin{aligned}
s_{\sigma \Omega^{2}}\left(q_{0}^{2}\right) & =2 \int_{0}^{1} \mathrm{~d} s_{1} q\left(s_{1}\right) \int_{0}^{1 / 2} \mathrm{~d} s_{2} \mathrm{e}^{\left|s_{2}-s_{1}\right| q_{0}^{2}} \\
& =4 d_{1 / 2}\left(q_{0}^{2}\right)-d_{1}\left(q_{0}^{2}\right),
\end{aligned}
$$

i.e., identical to the quadratic correlation function of the unconstrained diblock melt Eq. 24.

In order to determine the stability towards gelation, we have to consider the smaller eigenvalue of $G$, Eq. 35,

$$
\begin{aligned}
2 \lambda_{c}= & -\mu\left(1+8 d_{1 / 2}\left(q_{0}^{2}\right)\right) \\
& -\sqrt{\left[1+\mu\left(1-8 d_{1 / 2}\left(q_{0}^{2}\right)\right)\right]^{2}+\left[2 \mu_{\sigma_{0}} s_{\sigma \Omega^{2}}\left(q_{0}^{2}\right)\right]^{2}},
\end{aligned}
$$

and spot its change of sign. By consistently computing the lamellar amplitude $\sigma_{0}$ and wave number $q_{0}$ for each set $\left(\mu, L \chi_{\mu}\right)$ and the corresponding value of $\lambda_{c}$ and varying $L \chi$ until the latter approaches zero, we trace the lamellar gel instability $\mu_{c}(L \chi)$ shown as the dashed line in in Fig. 1. The end point toward small $\mu$ in this graph arises from the limitation of the approach to the vicinity of the fluid lamellar instability by the assumption that the amplitude $\sigma_{0}$ be small.

To summarize, the impact of microphase separation in the fluid is to shift the gelation transition to smaller critical cross-link chemical potentials. Microphase separation creates domains enriched in the selected component, so that cross-linking in the presence of $A B$ ordering is more efficient than without. This effect can be captured due to the cross-link ensemble, Eq. (9), which assigns a higher probability to network realizations with many node pairs close to each other in space at the instant of cross-linking.

\section{CONCLUSIONS AND OUTLOOK}

In this work, we have mapped out the phase states and transition lines in an $A B$ diblock copolymer melt crosslinked at random by irreversible and type-selective bonds. The central questions underlying this study were: How does random, type-selective cross-linking affect the chemical ordering transition? Vice versa, how does the ordering affect the gelation transition? Is there a novel transitions from the ordered fluid to the ordered gel? How do the different order parameters interact in a minimal field-theoretical description?

The resulting phase diagram is rather complex due to the symmetries of the underlying Hamiltonian. First, translational invariance is spontaneously broken in the gel state due to random localisation of particles. Secondly, considered superficially, microphase separation of symmetric diblocks is in the Ising universality class, and mean-field theory predicts a second-order phase transition. However, a more careful analysis of fluctuations has shown that microphase separation is instead in the Brazovskii universality class for weak crystallization ${ }^{2223}$ and exhibits a fluctuation-induced first-order transition 21 . Since our focus here has been on the coupling of microphase separation and gelation, we have restricted ourselves to the mean-field theory. Selective cross-linking gives rise to a partial pre-ordering into $A$ - and $B$-rich domains, resulting in a smaller critical $A B$ incompatibility $\chi_{c}$ for microphase separation. Still, the Brazovskil mechanism can be expected to be at work due to the shell of nonzero wavevectors which characterise the instability toward microphase separation also at these altered $\chi_{c}$. Further studies beyond mean field are needed to confirm this conjecture. Microphase separation, here into lamellae, facilitates the formation of a gel, because the success of cross-linking $A$-selectively is enhanced by the existence of $A$-rich domains (the same argument holds for $B$-selective cross-links). Therefore, fewer cross-links are needed to stabilise the network.

Several extensions of our work can and should be pursued. So far we have only considered isotropic localisation, which might be approximately valid for very weak segregation. However in general, localisation will be anisotropic, with strong localisation within the lamellar domains enriched in $A$ and weak localisation perpendicular to the lamellar plane. Our ansatz can be easily generalised to account for such anisotropies with different localisation lengths for localisation in plane and in between planes.

Based on the equilibrium analysis of this work, many striking features can be expected when turning to the nonequilibrium behavior. One especially interesting route to nonequilibrium is offered by the possibilty to cross-link the system at one temperature $\left(1 / \chi_{p}\right)$ and then measure its properties at a different temperature $\left(1 / \chi_{m}\right)$. For example, one can cross-link the sample within the lamellar phase, then decrease $\chi$ and study to what extent the system is able to (re)mix - in dependence on the cross-link density. Experimentally, it is possible to open cross-links by UV light, so that the system is allowed to partially relax, depending on the amount of opened cross-links. In general, phase ordering will be frustrated due to constraints imposed onto the system at a different degree of ordering. Yet, is it possible that a structure of frustrated order emerges when $\chi$ is increased in the disordered gel? Theoretically one can again use the Deam-Edwards distribution to allow for the analytical treatment of different states at preparation and mea- 
surement. Such studies have already been performed for cross-linked polymer blends $\sqrt{6}$, and work is in progress for the system considered here.

Another promising direction is to analyze the elastic properties of gel states with periodical composition modulation, in this study exemplified by lamellar gels. These states are expected to display special mechanical properties analogous to smectic liquid crystals, since the gel component is amorphous in all but one spatial direction, where the periodic composition modulation occurs. The difference is that the centres of mass in the gel component are randomly trapped such as in a glass. Topological constraints due to cross-linking may produce more complicated phase states or even phase coexistence in a block copolymer melt with sequence heterogeneity 24 .

Finally, as alluded to above, it would be desirable to go beyond mean-field theory, also to study coupled fluctuations of the two order parameters for localisation and for microphase separation. This is particularly interesting due to the drastic effects which fluctuations have already on microphase separation alone.

\section{ACKNOWLEDGMENTS}

Financial support of this work by the Deutsche Forschungsgemeinschaft through grant SFB-937/A4 is gratefully acknowledged. We thank K. Tietz, P. Vana, S. Finkhäuser, and K. Samwer from project A4 for the fruitful collaboration and M. Müller for interesting discussions.

\section{Appendix A: Route to the free-energy functional from the microscopic description}

Here, we sketch the calculation of the effective Hamiltonian or free-energy functional based on the microscopic model, which inter alia provides us with the explicit form of the vertices. First, we decouple the pair interactions quadratic in the collective densities, cf. Eqs. (2) and 5 , with (auxiliary) interaction fields $\tilde{\sigma}, \tilde{\varrho}$, and $\tilde{\Omega}$ using Hubbard-Stratonovich transforms. Then, we perform the average $[\cdot]_{\mathcal{P}_{\mathrm{DE}}}$ with the Deam-Edwards cross-link distribution Eq. (9) by means of the replica method 25 and obtain the replica partition function $\tilde{\mathcal{Z}}_{n+1}$. The latter involves $n+1$ replicas or system copies, since $\mathcal{P}_{\mathrm{DE}}$ requires an extra replica at preparation (labeled $\alpha=0$ in the following), in addition to the $n$ replicas at measurement (labeled $\alpha=1, \ldots, n$ ) usually employed to reformulate the average of $\ln \mathcal{Z}$. From $\tilde{\mathcal{Z}}_{n+1}$, the disorder-averaged free energy $[F]_{\mathcal{P}_{\mathrm{DE}}}$ follows as where $\lim _{n \rightarrow 0} \tilde{\mathcal{Z}}_{n+1}=\tilde{\mathcal{Z}}_{1}$.

$$
-[F]_{\mathcal{P}_{\mathrm{DE}}}=\lim _{n \rightarrow 0} \frac{\tilde{\mathcal{Z}}_{n+1}-\tilde{\mathcal{Z}}_{1}}{n \tilde{\mathcal{Z}}_{1}}
$$

$\tilde{\mathcal{Z}}_{n+1}=$

$\int \mathcal{D}\left[\tilde{\sigma}^{(\alpha)}, \tilde{\varrho}^{(\alpha)}\right] \int \mathcal{D}[\tilde{\Omega}] \exp \left\{-N \tilde{h}_{n+1}\left[\tilde{\sigma}^{(\alpha)}, \tilde{\varrho}^{(\alpha)}, \tilde{\Omega}\right]\right\}$.

Herein, $\tilde{\sigma}$ and $\tilde{\varrho}$ are, respectively, the interaction fields for the imbalance and the total density, confined to the 1 replica sector (1RS), and $\tilde{\Omega}$ the field interacting with the gel order parameter, which according to Eq. 113) has $\geq 2$ arguments $\boldsymbol{k}, \boldsymbol{p}, \ldots$ and thus is confined to the higher replica sector (HRS). The effective Hamiltonian which governs these field reads

$$
\begin{aligned}
& \tilde{h}_{n+1}= \\
& \frac{L}{4 \varrho_{0}^{2}} \sum_{\alpha=0}^{n} \sum_{\boldsymbol{k} \neq \mathbf{0}}\left[\chi_{\mu}^{(\alpha)} \tilde{\sigma}_{\boldsymbol{k}}^{(\alpha)} \tilde{\sigma}_{-\boldsymbol{k}}^{(\alpha)}+\kappa^{(\alpha)} \tilde{\varrho}_{\boldsymbol{k}}^{(\alpha)} \tilde{\varrho}_{-\boldsymbol{k}}^{(\alpha)}\right] \\
& +\frac{\mu}{2} \sum_{\hat{k} \in \mathrm{HRS}} \tilde{\Omega}_{\hat{k}} \tilde{\Omega}_{-\hat{k}}-\ln \tilde{z}_{n+1}\left[\tilde{\sigma}^{(\alpha)}, \tilde{\varrho}^{(\alpha)}, \tilde{\Omega}\right]
\end{aligned}
$$

with the single-polymer partition function

$$
\begin{aligned}
& \tilde{z}_{n+1}\left[\tilde{\sigma}^{(\alpha)}, \tilde{\varrho}^{(\alpha)}, \tilde{\Omega}\right]= \\
& \left\langle\exp \left\{\frac{1}{2 \varrho_{0}} \sum_{\alpha=0}^{n} \sum_{\boldsymbol{k} \neq \mathbf{0}} \int_{0}^{1} \mathrm{~d} s\left[L \chi_{\mu}^{(\alpha)} \tilde{\sigma}_{\boldsymbol{k}}^{(\alpha)}[q(s)-q]+i L \kappa^{(\alpha)} \tilde{\varrho}_{\boldsymbol{k}}^{(\alpha)}\right] \mathrm{e}^{-i \boldsymbol{k} \cdot \boldsymbol{r}^{(\alpha)}(s)}+2 \mu \sum_{\hat{k} \in \mathrm{HRS}} \tilde{\Omega}_{\hat{k}} \int_{0}^{1 / 2} \mathrm{~d} s \mathrm{e}^{-i \hat{k} \cdot \hat{r}(s)}\right\}\right\rangle_{\hat{\mathcal{H}}_{\mathrm{G}}}
\end{aligned}
$$

Hatted wave- and position vectors are used as a shorthand for arrays of $n+1$ vectors in replica space, $\hat{k}:=$ $\left(\boldsymbol{k}^{(0)}, \boldsymbol{k}^{(1)}, \ldots, \boldsymbol{k}^{(n)}\right)$, and $\hat{k} \cdot \hat{r}=\sum_{\alpha=0}^{n} \boldsymbol{k}^{(\alpha)} \cdot \boldsymbol{r}^{(\alpha)}$. Accordingly, here and in the following, $\langle\cdot\rangle_{\hat{\mathcal{H}}_{\mathrm{G}}}$ denotes the $n+1$-fold replicated single-chain conformational average. For the sake of transparency, we present the computation for $A B$-exchange symmetric melts, rigid cross-links $\left(a_{c} \rightarrow 0\right)$, and incompressibility. In this case, a sim- ple relation holds between the expectation values of the collective densities introduced in Sec. II A and of the interaction fields:

$$
\begin{aligned}
\left\langle\sigma_{\boldsymbol{k}}\right\rangle_{\mathcal{H}} & =\lim _{n \rightarrow 0}\left\langle\tilde{\sigma}_{\boldsymbol{k}}^{(\alpha)}\right\rangle_{N \tilde{h}_{n+1}}, \\
\left\langle\varrho_{\boldsymbol{k}}\right\rangle_{\mathcal{H}} & =i \lim _{n \rightarrow 0}\left\langle\tilde{\varrho}_{\boldsymbol{k}}^{(\alpha)}\right\rangle_{N \tilde{h}_{n+1}}
\end{aligned}
$$


(analogously for $\tilde{\Omega}$ ), so that on the saddle-point level we can identify these fields with the order parameters. Therefore, we return to the notation $\sigma, \varrho, \Omega$ for the fields. One further simplification in this case is that the elimination of total density fluctuations at the saddle point does not affect the instability towards phase separation.

\section{Appendix B: Vertices and saddle-point equations}

The complete set $s_{\sigma^{4}}$ of fourth-order vertices in $\sigma$ reads

$$
\begin{aligned}
\sum_{\boldsymbol{k}_{1}, \boldsymbol{k}_{2}, \boldsymbol{k}_{3}}^{\prime} s_{\sigma^{4}}= & \frac{L^{4}}{3 \cdot 2^{7} \varrho_{0}^{4}} \sum_{\alpha=0}^{n}\left(\chi_{\mu}^{(\alpha)}\right)^{4}\left\{\sum_{\boldsymbol{k}_{1}, \boldsymbol{k}_{2} \neq \mathbf{0}}\left[3 s\left(k_{1}^{2}\right) s\left(k_{2}^{2}\right)-s_{4}\left(\boldsymbol{k}_{1},-\boldsymbol{k}_{1}, \boldsymbol{k}_{2}\right)\right] \sigma_{\boldsymbol{k}_{1}}^{(\alpha)} \sigma_{-\boldsymbol{k}_{1}}^{(\alpha)} \sigma_{\boldsymbol{k}_{2}}^{(\alpha)} \sigma_{-\boldsymbol{k}_{2}}^{(\alpha)}\right. \\
& +\sum_{\boldsymbol{k}_{1} \neq-\boldsymbol{k}_{2}, \boldsymbol{k}_{3}}^{\prime}\left[3 \frac{s_{\sigma^{2} \varrho}\left(\boldsymbol{k}_{1}, \boldsymbol{k}_{2}\right) s_{\sigma^{2} \varrho}\left(\boldsymbol{k}_{3},-\sum_{\mathrm{r}=1}^{3} \boldsymbol{k}_{\mathrm{r}}\right)}{d_{1}\left(\left(\boldsymbol{k}_{1}+\boldsymbol{k}_{2}\right)^{2}\right)}-s_{4}\left(\boldsymbol{k}_{1}, \boldsymbol{k}_{2}, \boldsymbol{k}_{3}\right)\right] \sigma_{\boldsymbol{k}_{1}}^{(\alpha)} \sigma_{\boldsymbol{k}_{2}}^{(\alpha)} \sigma_{\boldsymbol{k}_{3}}^{(\alpha)} \sigma_{-\sum_{\mathrm{r}=1}^{(\alpha)} \boldsymbol{k}_{\mathrm{r}}}^{(\alpha)}
\end{aligned}
$$

with the correlation function $s_{\sigma^{2}} \varrho$ due to the contribution of generalized density modulations,

$$
\begin{aligned}
& s_{\sigma^{2} \varrho}\left(\boldsymbol{k}_{1}, \boldsymbol{k}_{2}\right) \\
& :=\iiint_{0}^{1} \mathrm{~d} s_{1} \mathrm{~d} s_{2} \mathrm{~d} s_{3} q\left(s_{1}\right) q\left(s_{2}\right) \\
& \times\left\langle\mathrm{e}^{-i\left(\boldsymbol{k}_{1} \cdot \boldsymbol{r}^{(\alpha)}\left(s_{1}\right)+\boldsymbol{k}_{2} \cdot \boldsymbol{r}^{(\alpha)}\left(s_{2}\right)+\boldsymbol{k}_{3} \cdot \boldsymbol{r}^{(\alpha)}\left(s_{3}\right)\right)}\right\rangle_{\hat{\mathcal{H}}_{\mathrm{G}}} \\
& =\iiint_{0}^{1} \mathrm{~d} s_{1} \mathrm{~d} s_{2} \mathrm{~d} s_{3} q\left(s_{1}\right) q\left(s_{2}\right) \\
& \times \mathrm{e}^{\left|s_{2}-s_{1}\right| \boldsymbol{k}_{1} \cdot \boldsymbol{k}_{2}-\left|s_{3}-s_{2}\right|\left(\boldsymbol{k}_{1} \cdot \boldsymbol{k}_{2}+k_{2}^{2}\right)-\left|s_{3}-s_{1}\right|\left(\boldsymbol{k}_{1} \cdot \boldsymbol{k}_{2}+k_{1}^{2}\right)}
\end{aligned}
$$

(this function has been computed as $s^{(\alpha)}\left(\boldsymbol{k}_{1}, \boldsymbol{k}_{2}\right)$ in 26 , also for diblocks). The explicit form of the fourth-order correlation $s_{4}\left(\boldsymbol{k}_{1}, \boldsymbol{k}_{2}, \boldsymbol{k}_{3}\right)$ evaluated for lamellar phase separation and for symmetric diblocks can be found in 26 , denoted as $s^{(\beta)}$.

The general saddle-point equation which determines the second moment of the gel order parameter as a function of the order parameter for microphase separation is

$$
\overline{\Omega_{\left(\boldsymbol{k}_{1}, \boldsymbol{k}_{2}\right)}}=\frac{\left(L \chi_{\mu}\right)^{2} s_{\sigma^{2} \Omega}\left(k_{1}^{2}, k_{2}^{2}\right) \overline{\sigma_{\boldsymbol{k}_{1}}} \overline{\sigma_{\boldsymbol{k}_{2}}}}{8 \varrho_{0}^{2}\left(1-\mu \overline{d_{1 / 2}\left(k_{1}^{2}+k_{2}^{2}\right)}\right)}+\mathcal{O}\left(|\sigma|^{3}\right)
$$

where we assumed replica-symmetry. The correlation function $s_{\sigma^{2} \Omega}\left(k_{1}^{2}, k_{2}^{2}\right)$ in Eq. (B3), first appearing in Eq. 27), for $\hat{k}=\boldsymbol{k}_{1} \hat{e}^{(\alpha)}+\boldsymbol{k}_{1} \hat{e}^{(\beta)}$ and $\alpha \neq \beta$ is

$$
\begin{aligned}
s_{\sigma^{2} \Omega} & \left(k_{1}^{2}, k_{2}^{2}\right) \\
:= & 2 \iint_{0}^{1} \mathrm{~d} s_{1} \mathrm{~d} s_{2} q\left(s_{1}\right) q\left(s_{2}\right) \int_{s_{3}=0}^{1 / 2} \mathrm{~d} s_{3} \\
& \times\left\langle\mathrm{e}^{-i\left(\boldsymbol{k}_{1} \cdot \boldsymbol{r}^{(\alpha)}\left(s_{1}\right)+\boldsymbol{k}_{2} \cdot \boldsymbol{r}^{(\beta)}\left(s_{2}\right)+\hat{k} \cdot \hat{r}\left(s_{3}\right)\right)}\right\rangle_{\hat{\mathcal{H}}_{\mathrm{G}}} \\
= & 2 \iint_{0}^{1} \mathrm{~d} s_{1} \mathrm{~d} s_{2} q\left(s_{1}\right) q\left(s_{2}\right) \int_{s_{3}=0}^{1 / 2} \mathrm{~d} s_{3} \\
& \times \mathrm{e}^{-\left|s_{1}-s_{3}\right| k_{1}^{2}-\left|s_{2}-s_{3}\right| k_{2}^{2}} .
\end{aligned}
$$

With the contributions of the generalized density modulations and the gel order parameter, the fluid lamellar amplitude is determined by

$$
\begin{aligned}
& \left(\frac{L \chi_{\mu} \sigma_{0}}{4 \varrho_{0}}\right)^{2}=\Phi\left(q_{m}\right)=\max _{k} \Phi(k), \\
& \Phi(k):=\frac{\frac{s\left(k^{2}\right)}{2}-\frac{1}{L \chi_{\mu}}}{D\left(k^{2}\right)}, \\
& L \chi_{\mu} \geq L \chi_{\mu, c}=\frac{2}{s\left(q_{0}^{2}\right)}, \quad \mu<1, \quad q_{\mathrm{m}}:=\underset{k}{\operatorname{argmax}} \Phi(k),
\end{aligned}
$$

with the denominator

$$
\begin{aligned}
D\left(k^{2}\right)= & 2\left(s\left(k^{2}\right)\right)^{2}+\frac{\left(s_{\sigma^{2} \varrho}(k \boldsymbol{n}, k \boldsymbol{n})\right)^{2}}{d_{1}\left(4 k^{2}\right)} \\
& -s_{4}(k \boldsymbol{n}, k \boldsymbol{n},-k \boldsymbol{n})-2 \mu \frac{\left(s_{\sigma^{2} \Omega}\left(k^{2}, k^{2}\right)\right)^{2}}{1-4 \mu d_{1 / 2}\left(2 k^{2}\right)},
\end{aligned}
$$

and $\boldsymbol{n}$ the arbitrary, but fixed unit normal defined by $\boldsymbol{q}_{0}$.

The other correlation function in Eq. 27. is

$$
\begin{aligned}
& s_{\sigma \Omega^{2}}\left(\boldsymbol{k}^{(\alpha)} ; \hat{k}\right)= \\
& 4 \int_{0}^{1} \mathrm{~d} s_{1} q\left(s_{1}\right) \iint_{0}^{1 / 2} \mathrm{~d} s_{2} \mathrm{~d} s_{3} \\
& \quad \times \mathrm{e}^{\left|s_{2}-s_{1}\right| \boldsymbol{k}^{(\alpha)} \cdot \hat{k}-\left|s_{3}-s_{1}\right|\left(\boldsymbol{k}^{(\alpha)} \cdot \hat{k}+k^{2}\right)-\left|s_{3}-s_{2}\right|\left(\boldsymbol{k}^{(\alpha)} \cdot \hat{k}+\hat{k}^{2}\right)},
\end{aligned}
$$

and is essential to determine the instability toward the lamellar gel in Sec. VI.

The third-order correlation for the gel order parameter in Eq. 26) is

$$
\begin{aligned}
s_{\Omega^{3}}\left(\hat{k}_{1}, \hat{k}_{2}\right)= & 8 \iiint_{0}^{1 / 2} \mathrm{~d} s_{1} \mathrm{~d} s_{2} \mathrm{~d} s_{3} \mathrm{e}^{\left|s_{2}-s_{1}\right| \hat{k}_{1} \cdot \hat{k}_{2}} \\
& \times \mathrm{e}^{-\left|s_{3}-s_{1}\right|\left(\hat{k}_{1} \cdot \hat{k}_{2}+\hat{k}_{1}^{2}\right)-\left|s_{3}-s_{2}\right|\left(\hat{k}_{1} \cdot \hat{k}_{2}+\hat{k}_{2}^{2}\right)} .
\end{aligned}
$$


${ }^{1}$ L. Leibler, Macromolecules 13, 1602 (1980).

${ }^{2}$ F. S. Bates and G. H. Fredrickson, Annu. Rev. Phys. Chem. 41, 525 (1990).

${ }^{3}$ M. W. Matsen and M. Schick, Phys. Rev. Lett. 72, 2660 (1994).

${ }^{4}$ A. K. Chakraborty, Phys. Rep. 342, 1 (2001).

${ }^{5}$ C. D. Sfatos and E. I. Shakhnovich, Phys. Rep. 288, 77 (1997).

${ }^{6}$ C. Wald, A. Zippelius, and P. M. Goldbart, Europhys. Lett. 70, 843 (2005).

${ }^{7}$ C. Wald, P. M. Goldbart, and A. Zippelius, J. Chem. Phys. 124, 214905 (2006).

${ }^{8}$ R. E. Burge, A. G. Fowler, and D. A. Reaveley, J. Mol. Biol. 117, 927 (1977).

${ }^{9}$ H. J. Rogers, H. R. Perkins, and J. B. Ward, Microbial cell walls and membranes (Chapman and Hall, London, 1980).

${ }^{10}$ P. M. Goldbart, H. Castillo, and A. Zippelius, Adv. Phys. 45, 393 (1996).

${ }^{11}$ S. V. Panyukov and Y. Rabin, Physics Reports 269, 1 (1996).

${ }^{12}$ K. Tietz, S. Finkhäuser, K. Samwer, and P. Vana, Macromol. Chem. Phys. 215, 1563 (2014).

${ }^{13}$ R. T. Deam and S. F. Edwards, Philos. Trans. R. Soc. London Ser. A 280, 317 (1976).

${ }^{14}$ E. D. Gomez, J. Das, A. K. Chakraborty, J. A. Pople, and N. P. Balsara, Macromolecules 39, 4848 (2006).
${ }^{15}$ S. Lay, J.-U. Sommer, and A. Blumen, J. Chem. Phys. 113, 11355 (2000).

${ }^{16}$ S. Lay, J.-U. Sommer, and A. Blumen, J. Chem. Phys. 110, 12173 (1999).

${ }^{17}$ P. J. Flory, J. Am. Chem. Soc. 63, 3083, 3091, 3096 (1941).

${ }^{18}$ W. H. Stockmayer, J. Chem. Phys. 11, 45 (1943).

${ }^{19}$ S. Ulrich, Ph.D. thesis, Univ. Göttingen (2010).

${ }^{20}$ M. Huthmann, M. Rehkopf, A. Zippelius, and P. M. Goldbart, Phys. Rev. E 54, 3943 (1996).

${ }^{21}$ G. H. Fredrickson and E. Helfand, J. Chem. Phys. 87, 697 (1987).

${ }^{22}$ S. A. Brazovskiı̌, Zh. Eksp. Teor. Fiz. [Sov. Phys. JETP] 68 [41], 175 [85] (1975).

${ }^{23}$ S. A. Brazovskǐr, I. E. Dzyaloshinskiŭ, and A. R. Muratov, Sov. Phys. JETP 66, 625 (1987).

${ }^{24}$ A. von der Heydt, M. Müller, and A. Zippelius, Phys. Rev. E 83, 051131 (2011).

${ }^{25}$ M. Mézard, G. Parisi, and M. A. Virasoro, Spin Glass Theory and Beyond, Lecture Notes in Physics, Vol. 9 (World Scientific, Singapore, 1987).

${ }^{26}$ A. von der Heydt, Ph.D. thesis Univ. Göttingen (2011). 